\title{
Cone beam computed tomography: An accurate imaging technique in comparison with orthogonal portal imaging in intensity-modulated radiotherapy for prostate cancer
}

\author{
Om Prakash Gurjar ${ }^{1}$, Abhinav Mutneja ${ }^{1}$, Priyusha Bagdare ${ }^{1}$, Harsh Goyal' ${ }^{1}$, Manika Batra ${ }^{1}$, \\ Virendra Bhandari', Krishna Lal Gupta1, Surendra Prasad Mishra²
}

${ }^{1}$ Roentgen-SAIMS Radiation Oncology Centre, Sri Aurobindo Institute of Medical Sciences, Indore, Madhya Pradesh, India ${ }^{2}$ Department of Radiotherapy, Dr. Ram Manohar Lohia Institute of Medical Sciences, Lucknow, India

Received September 30, 2015; Revised January 17, 2016; Accepted January 23, 2016; Published Online February 03, 2016

Original Article

\begin{abstract}
Purpose: Various factors cause geometric uncertainties during prostate radiotherapy, including interfractional and intrafractional patient motions, organ motion, and daily setup errors. This may lead to increased normal tissue complications when a high dose to the prostate is administered. More-accurate treatment delivery is possible with daily imaging and localization of the prostate. This study aims to measure the shift of the prostate by using kilovoltage (kV) cone beam computed tomography (CBCT) after position verification by $\mathrm{kV}$ orthogonal portal imaging (OPI). Methods: Position verification in 10 patients with prostate cancer was performed by using OPI followed by CBCT before treatment delivery in 25 sessions per patient. In each session, OPI was performed by using an on-board imaging (OBI) system and pelvic bone-to-pelvic bone matching was performed. After applying the noted shift by using OPI, CBCT was performed by using the OBI system and prostate-to-prostate matching was performed. The isocenter shifts along all three translational directions in both techniques were combined into a three-dimensional (3-D) iso-displacement vector (IDV). Results: The mean (SD) IDV (in centimeters) calculated during the 250 imaging sessions was 0.931 (0.598, median 0.825 ) for OPI and 0.515 (336, median 0.43 ) for CBCT, $p$-value was less than 0.0001 which shows extremely statistical significant difference. Conclusion: Even after bone-to-bone matching by using OPI, a significant shift in prostate was observed on CBCT. This study concludes that imaging with CBCT provides a more accurate prostate localization than the OPI technique. Hence, CBCT should be chosen as the preferred imaging technique.
\end{abstract}

Keywords: Cone Beam Computed Tomography; Organ Motion; Orthogonal Portal Imaging; Prostate Cancer

\section{Introduction}

Prostate cancer is the second most common type of cancer and the fifth leading cause of cancer-related death in men worldwide. ${ }^{1}$ Prostate cancer can be managed by using surgery, chemotherapy, radiotherapy, and so on. Among these treatment options, radiotherapy is considered superior in terms of survival rate and side effects. Three-dimensional conformal radiotherapy (3-DCRT), intensity-modulated radiotherapy (IMRT), imaging-guided radiotherapy (IGRT), volumetric

modulated arc therapy (VMAT) and intensity modulated proton therapy (IMPT) are various modern techniques by which prostate cancer can be treated.2,3 All these modern radiotherapy techniques basically focus on lethal-dose delivery to the target volume and minimum dose delivery to the organs at risk (OAR). ${ }^{4}$

In IMRT, propinquity between the prostate and the OAR such as the bladder and rectum often leads to highly 
inhomogeneous fluence profiles, with steep dose gradients in concave, tumor wrapped around the OAR or in convex structures. In addition, in the case of prostate cancer, many factors cause geometric uncertainties during prostate radiotherapy, including interfractional and intrafractional patient motions, organ motion, and daily setup errors. ${ }^{5}$ This may lead to increased complications in OAR when a high dose to the prostate is administered. Hence, more-accurate treatment delivery is possible with proper patient immobilization and accurate setup verification by using the adequate imaging technique before delivering each fraction.

Published studies demonstrate that the tumor control probability (TCP) and toxicity of OAR are closely associated with proper treatment execution. Radiotherapy with better accuracy is sufficient to improve the TCP and decrease treatment-related morbidity. ${ }^{6}$ Bentel et al. ${ }^{7}$ demonstrated the effectiveness of immobilization and emphasized the requirements for improving immobilizing techniques. Mounting the on-board imaging (OBI) system on the gantry of a medical electron linear accelerator (Clinac) is efficient to verify patient setup accuracy and position reproducibility.,9 Earlier study done on comparison between kilovoltage $(\mathrm{kV})$ orthogonal portal imaging (OPI) and kV-cone beam computed tomography (CBCT) imaging for various sites viz. brain, head-and-neck and pelvis shows that OPI is enough in the case of brain and head-and-neck cases as the target volume doesn't move significantly with respect to bones in such sites, however the study recommended that CBCT should be preferred in the case of sites with movable targets e.g. prostate cancer. ${ }^{9}$ In general, daily imaging is advised in the case of prostate cancer and is considered as the standard practice. ${ }^{10,11}$ However, in many centers, daily imaging is not performed even in the case of moving targets such as prostate cancers owing to the unavailability of imaging techniques or high patient workload, and many centers implement a protocol for daily portal imaging.

The OBI system consists of two kinds of imaging techniques, namely $\mathrm{kV}-\mathrm{OPI}$ and $\mathrm{kV}$-CBCT. In this study, the position verification with the OPI technique was cross-verified by using the CBCT technique.

\section{Methods and Materials}

A prospective study was conducted from July 2014 to January 2015 with 10 patients with prostate cancer who were scheduled for imaging IGRT at a dose of $50 \mathrm{~Gy}$ in 25 fractions (\#) at the rate of $2 \mathrm{~Gy} / \#$ in the first treatment phase. Although a total dose of $78 \mathrm{~Gy} / 38 \#$ was planned and delivered in the first, second, and third phases of the treatment plan, this study was conducted during the first treatment phase only. Patients with histologically confirmed early-stage prostate cancer, except patients with nodal metastasis, were selected for the study. The age of the patients ranged from 50 to 80 years. In this study, the accuracy of the position verification with the OPI technique was cross-verified by using the CBCT technique. With the OPI technique, 250 imaging sessions (10 patients $\times 25 \#)$ were performed. Same person was involved in the imaging procedures for all the patients and imaging sessions to avoid the person to person differences.

Full bladder protocol was followed by most of the patients and each patient was suggested for urine pass and then to drink $200 \mathrm{ml}$ of water 30 minutes before taking on the couch in each session. However, few patients did not follow this protocol due to their critical clinical problems.

For all of the patients, six clamp thermoplastic sheets (Orfit) for the immobilization of the pelvic region were molded in the molding room. Then, computed tomography (CT) was performed by using the Siemens SOMATOM Definition AS scanner (Siemens Medical Systems, Germany). The CT images of 3-mm-thick slices were acquired and transferred to the treatment planning system (TPS) Eclipse version 8.9 (Varian Medical Systems, Palo Alto, CA). Gross tumor volume (GTV), clinical target volume (CTV), planning target volume (PTV), and OAR were delineated on the CT images, following the guidelines of the International Commission on Radiation Units and Measurements report No. 83 (ICRU 83). ${ }^{12}$ PTV margin of $0.5 \mathrm{~cm}$ around the CTV was given in all the cases. IGRT plans were created with a 6-MV photon beam, and Varian leaf motion calculator version 8.9.08 was utilized to calculate leaf motion for dynamic dose delivery. A dose volume optimizer (DVO) was used for planning optimization. Anisotropic analytical algorithm (AAA) was used to calculate doses with a grid size of $0.25 \mathrm{cc}$. After approval, the plans were scheduled for 25 fractions with daily imaging with the OPI and CBCT techniques.

The patients were positioned and immobilized on the couch of the Varian Clinac DMX (Varian Medical Systems, Palo Alto, CA) by using the thermoplastic sheet molded for them. Then, OPI and CBCT were performed with the OBI system (Varian Medical Systems, Palo Alto, CA) consisting of a $125-\mathrm{kV}_{\mathrm{p}}$ x-ray tube isocentrically mounted to the gantry of the Clinac. It was operated from the treatment console. Anatomy-matching software (Varian Portal Vision 7.5) was used to study the patient setup deviations and to determine the spatial coordinates in the images. The ARIA networking system (Varian Medical Systems Inc.) was used to upload the reference images from the TPS.

Before each treatment session, OPI was performed with an imager vertical distance of $50 \mathrm{~cm}$, where two portal images were taken at gantry angles of $0^{\circ}$ and $90^{\circ}$, respectively. The portal images were superimposed on reference digitally reconstructed radiograph (DRR) images, and two pelvic bones were matched by using the 
spyglass technique as shown in Figure 1 (a), in which a movable inner window separated the reference DRR on the inside from the OPI image on the outside. The encompass is appraised by examining the continuity of the bones across the edges of the inner window. ${ }^{13}$ The isocenter shifts along three translational directions, namely the lateral, longitudinal, and vertical directions, were noted and applied to the Clinac couch.

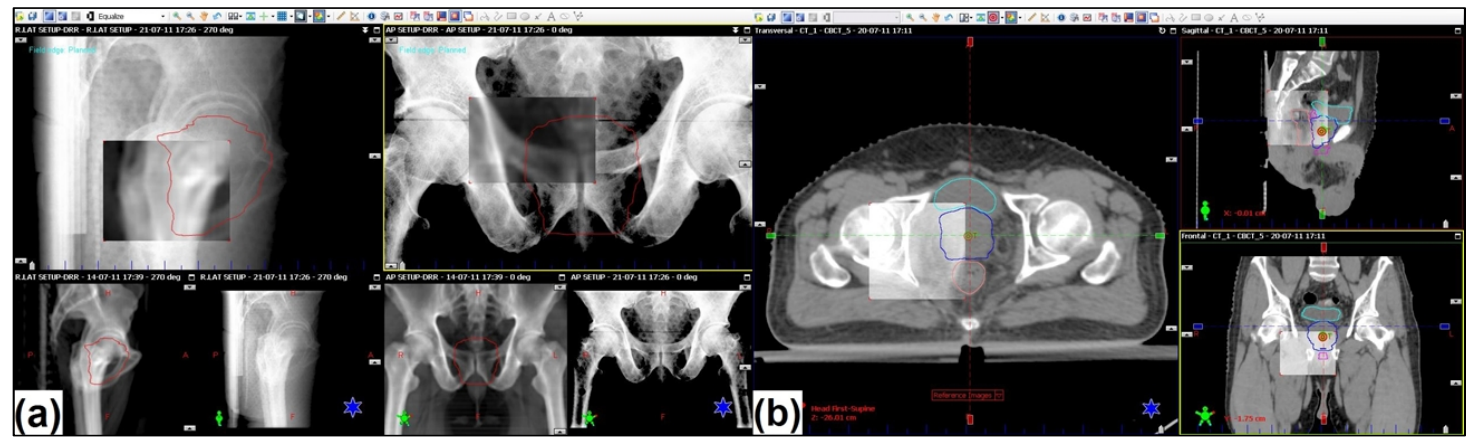

Figure 1: Registration window of the on-board imaging system (OBI) for (a) orthogonal portal imaging (OPI) and (b) cone beam computed tomography (CBCT).

As the prostate is a soft tissue organ whose position may vary according to the position of the bones, to cross-verify the matching performed by using the OPI technique, CBCT was performed at an imager vertical distance of $50 \mathrm{~cm}$ and by using a full-bowtie filter. CBCT was performed by rotating the gantry in the counterclockwise direction from $179^{\circ}$ to $180^{\circ}\left(360^{\circ}\right.$ rotation). The CBCT images were fused with the reference CT images, and two prostates were matched as shown in Figure 1 (b), with the help of transversal, frontal, and sagittal views by using the above-mentioned spyglass method. The isocenter shifts along the three translational directions were noted and applied to the Clinac couch.

In the two above-mentioned techniques, rotational shift was not considered. However, it was very small $\left(<1^{\circ}\right)$ in few imaging sessions. The shifts in all the three translational directions in each of the 250 sessions for both techniques were noted and analyzed. The mean values and standard deviation (SD) were calculated. The isocenter shifts along all the three translational directions in both techniques were combined in to a 3-D isodisplacement vector (IDV), which is defined as $E=$ $\left(\text { vert }^{2}+\text { long }^{2}+\text { lat }^{2}\right)^{1 / 2} \cdot{ }^{9}$ Paired $t$-test was applied to calculate the $p$-value for the statistical analysis of the difference between IDV calculated in OPI and CBCT techniques.

\section{Results}

Patient position verification before each of the 250 treatment sessions (10 patients $\times 25 \#)$ was performed by using OPI. The mean (SD) [median] shifts (in centimeters) were $0.230(0.261)$ [0.2], 0.792 (0.628) [0.7], and $0.212(0.190)$ [0.1] in the vertical, longitudinal, and lateral directions, respectively. To cross-verify the position, CBCT was performed, and the mean (SD) [median] shifts (in centimeters) were 0.125 (0.117) [0.1], $0.425(0.331)$ [0.3], and $0.199(0.139)$ [0.2] in the vertical, longitudinal, and lateral directions, respectively. The shifts in all the three translational directions in the 250 imaging sessions that were measured by using OPI and CBCT are depicted in Figures 2 and 3, respectively. The obtained setup errors in this study are systematic and random errors for the currently applied treatment techniques, using basic immobilization devices.

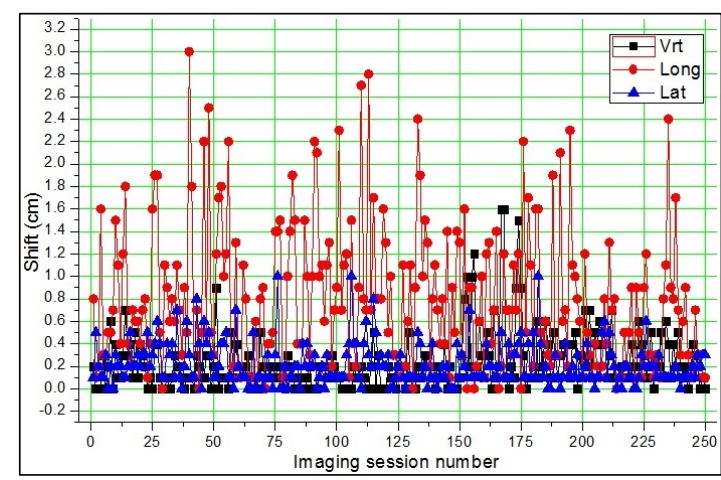

Figure 2: The shifts in all the three translational directions, namely the vertical (Vrt), longitudinal (Long), and lateral (Lat) directions, from the 250 imaging sessions that were measured by using orthogonal portal imaging.

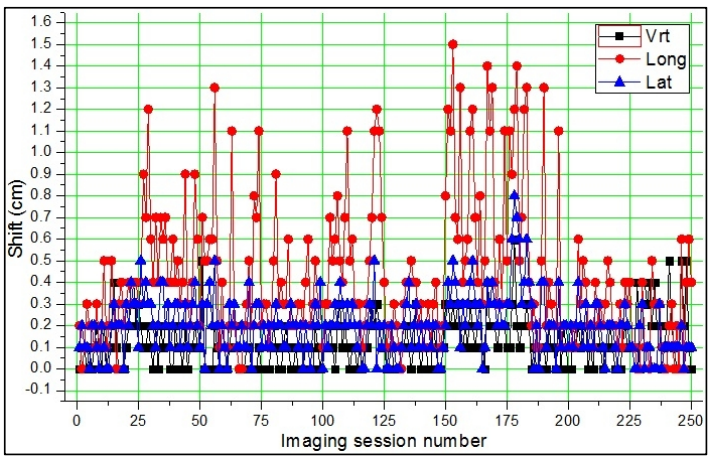

Figure 3: The shifts in all the three translational directions, namely the vertical (Vrt), longitudinal (Long), and lateral (Lat) directions, from the 250 imaging sessions that were measured by using cone beam computed tomography. 
The mean (SD) [median] IDV (in centimeters) calculated during the 250 imaging sessions was 0.931 (0.598) [0.825] for OPI and 0.515 (336) [0.43] for CBCT, respectively. The calculated $p$-value was less than 0.0001 which shows the extremely statistical significant difference between the IDV calculated in both the techniques.
Table 1 shows the mean IDV for each of the 10 patients that was calculated by using the shifts in all the three translational directions measured by using OPI and CBCT.

Table 1: Measured shifts, calculated isodisplacement vector (IDV), standard deviation (SD), and median values for 10 patients with prostate cancer.

\begin{tabular}{|c|c|c|c|c|c|c|c|c|c|}
\hline \multirow{2}{*}{$\begin{array}{l}\text { Patient } \\
\text { no. }\end{array}$} & \multirow{2}{*}{$\begin{array}{l}\text { No. of } \\
\text { imaging } \\
\text { sessions }\end{array}$} & \multicolumn{4}{|c|}{ OPI } & \multicolumn{4}{|c|}{ CBCT } \\
\hline & & $\begin{array}{l}\text { Mean vrt. } \\
\text { Shift in } \mathrm{cm} \\
(\mathrm{SD})\end{array}$ & $\begin{array}{l}\text { Mean } \\
\text { long. Shift } \\
\text { in } \mathrm{cm}(\mathrm{SD})\end{array}$ & $\begin{array}{l}\text { Mean lat. } \\
\text { Shift in } \\
\text { cm (SD) }\end{array}$ & $\begin{array}{l}\text { Mean } \\
\text { IDV in } \\
\text { cm (SD) }\end{array}$ & $\begin{array}{l}\text { Mean vrt. } \\
\text { Shift in } \\
\text { cm (SD) }\end{array}$ & $\begin{array}{l}\text { Mean } \\
\text { long. Shift } \\
\text { in } \mathrm{cm}(\mathrm{SD})\end{array}$ & $\begin{array}{l}\text { Mean lat. } \\
\text { Shift in } \\
\text { cm (SD) }\end{array}$ & $\begin{array}{l}\text { Mean } \\
\text { IDV in } \\
\mathrm{cm}(\mathrm{SD})\end{array}$ \\
\hline 1 & 25 & $\begin{array}{c}0.212 \\
(0.203)\end{array}$ & $\begin{array}{c}0.700 \\
(0.496)\end{array}$ & $\begin{array}{c}0.224 \\
(0.136)\end{array}$ & $\begin{array}{c}0.832 \\
(0.441)\end{array}$ & $\begin{array}{c}0.128 \\
(0.124)\end{array}$ & $\begin{array}{c}0.252 \\
(0.139)\end{array}$ & $\begin{array}{c}0.160 \\
(0.112)\end{array}$ & $\begin{array}{c}0.355 \\
(0.161)\end{array}$ \\
\hline 2 & 25 & $\begin{array}{c}0.148 \\
(0.126)\end{array}$ & $\begin{array}{c}0.956 \\
(0.804)\end{array}$ & $\begin{array}{c}0.320 \\
(0.227)\end{array}$ & $\begin{array}{c}1.069 \\
(0.778)\end{array}$ & $\begin{array}{c}0.112 \\
(0.078)\end{array}$ & $\begin{array}{c}0.552 \\
(0.245)\end{array}$ & $\begin{array}{c}0.256 \\
(0.112)\end{array}$ & $\begin{array}{c}0.632 \\
(0.249)\end{array}$ \\
\hline 3 & 25 & $\begin{array}{c}0.192 \\
(0.204)\end{array}$ & $\begin{array}{c}0.732 \\
(0.628)\end{array}$ & $\begin{array}{c}0.18 \\
(0.189)\end{array}$ & $\begin{array}{c}0.844 \\
(0.601)\end{array}$ & $\begin{array}{c}0.068 \\
(0.114)\end{array}$ & $\begin{array}{c}0.440 \\
(0.359)\end{array}$ & $\begin{array}{c}0.196 \\
(0.137)\end{array}$ & $\begin{array}{c}0.514 \\
(0.364)\end{array}$ \\
\hline 4 & 25 & $\begin{array}{c}0.116 \\
(0.099)\end{array}$ & $\begin{array}{c}1.004 \\
(0.603)\end{array}$ & $\begin{array}{c}0.180 \\
(0.208)\end{array}$ & $\begin{array}{c}1.061 \\
(0.585)\end{array}$ & $\begin{array}{c}0.060 \\
(0.071)\end{array}$ & $\begin{array}{c}0.332 \\
(0.177)\end{array}$ & $\begin{array}{c}0.172 \\
(0.098)\end{array}$ & $\begin{array}{c}0.397 \\
(0.178)\end{array}$ \\
\hline 5 & 25 & $\begin{array}{c}0.096 \\
(0.124)\end{array}$ & $\begin{array}{c}1.012 \\
(0.746)\end{array}$ & $\begin{array}{c}0.288 \\
(0.244)\end{array}$ & $\begin{array}{c}1.110 \\
(0.715)\end{array}$ & $\begin{array}{c}0.132 \\
(0.090)\end{array}$ & $\begin{array}{c}0.568 \\
(0.305)\end{array}$ & $\begin{array}{c}0.204 \\
(0.117)\end{array}$ & $\begin{array}{c}0.632 \\
(0.311)\end{array}$ \\
\hline 6 & 25 & $\begin{array}{c}0.136 \\
(0.132)\end{array}$ & $\begin{array}{c}0.884 \\
(0.593)\end{array}$ & $\begin{array}{c}0.148 \\
(0.145)\end{array}$ & $\begin{array}{c}0.942 \\
(0.567)\end{array}$ & $\begin{array}{c}0.084 \\
(0.089)\end{array}$ & $\begin{array}{c}0.264 \\
(0.170)\end{array}$ & $\begin{array}{c}0.140 \\
(0.108)\end{array}$ & $\begin{array}{c}0.330 \\
(0.190)\end{array}$ \\
\hline 7 & 25 & $\begin{array}{c}0.576 \\
(0.514)\end{array}$ & $\begin{array}{c}0.668 \\
(0.498)\end{array}$ & $\begin{array}{c}0.232 \\
(0.168)\end{array}$ & $\begin{array}{c}1.046 \\
(0.517)\end{array}$ & $\begin{array}{c}0.196 \\
(0.089)\end{array}$ & $\begin{array}{c}0.796 \\
(0.391)\end{array}$ & $\begin{array}{c}0.288 \\
(0.120)\end{array}$ & $\begin{array}{c}0.881 \\
(0.392)\end{array}$ \\
\hline 8 & 25 & $\begin{array}{c}0.272 \\
(0.184)\end{array}$ & $\begin{array}{c}0.916 \\
(0.703)\end{array}$ & $\begin{array}{c}0.200 \\
(0.216)\end{array}$ & $\begin{array}{c}1.033 \\
(0.676)\end{array}$ & $\begin{array}{c}0.144 \\
(0.133)\end{array}$ & $\begin{array}{c}0.564 \\
0.467)\end{array}$ & $\begin{array}{c}0.308 \\
(0.222)\end{array}$ & $\begin{array}{c}0.683 \\
(0.500)\end{array}$ \\
\hline 9 & 25 & $\begin{array}{c}0.324 \\
(0.217)\end{array}$ & $\begin{array}{c}0.472 \\
(0.374)\end{array}$ & $\begin{array}{c}0.180 \\
(0.168)\end{array}$ & $\begin{array}{c}0.658 \\
(0.373)\end{array}$ & $\begin{array}{c}0.104 \\
(0.079)\end{array}$ & $\begin{array}{c}0.280 \\
(0.141)\end{array}$ & $\begin{array}{c}0.180 \\
(0.096)\end{array}$ & $\begin{array}{c}0.364 \\
(0.154)\end{array}$ \\
\hline 10 & 25 & $\begin{array}{c}0.228 \\
(0.179) \\
\end{array}$ & $\begin{array}{c}0.580 \\
(0.569) \\
\end{array}$ & $\begin{array}{c}0.172 \\
(0.121) \\
\end{array}$ & $\begin{array}{c}0.715 \\
(0.523) \\
\end{array}$ & $\begin{array}{c}0.220 \\
(0.173) \\
\end{array}$ & $\begin{array}{c}0.200 \\
(0.198) \\
\end{array}$ & $\begin{array}{c}0.092 \\
(0.086) \\
\end{array}$ & $\begin{array}{c}0.365 \\
(0.198) \\
\end{array}$ \\
\hline \multicolumn{2}{|c|}{$\begin{array}{l}\text { Mean of } 250 \\
\text { imaging sessions }\end{array}$} & 0.230 & 0.792 & 0.212 & 0.931 & 0.125 & 0.425 & 0.199 & 0.515 \\
\hline \multicolumn{2}{|c|}{$\begin{array}{l}\text { SD of } 250 \text { imaging } \\
\text { sessions }\end{array}$} & 0.261 & 0.628 & 0.190 & 0.598 & 0.117 & 0.331 & 0.139 & 0.336 \\
\hline \multicolumn{2}{|c|}{$\begin{array}{l}\text { Median of } 250 \\
\text { imaging sessions }\end{array}$} & 0.200 & 0.700 & 0.100 & 0.825 & 0.100 & 0.300 & 0.200 & 0.430 \\
\hline
\end{tabular}

\section{Discussion}

This was a prospective study conducted to investigate the efficacy of OPI as the imaging technique during prostate cancer radiotherapy by cross-verification by using the CBCT technique. The scope of OPI is limited to the set-up corrections utilizing bony landmarks. It is a well-known fact that for sites that have limited moving structures such as the brain and the head-and-neck region, utilizing bony information is adequate for setup error correction for advanced radiotherapy treatments. OBI was introduced because of its potential to visualize 3-D soft tissue anatomy. CBCT image acquisition is based on a large number of projections and thus is likely to provide superior quantitative spatial information over that provided by a planar $\mathrm{kV}$ image acquisition using OPI.

The imaging results obtained by using OPI concurred with the results of the published studies on daily imaging for prostate cancer radiotherapy. ${ }^{14,15}$ The setup errors in this technique include the systematic and random errors as the basic immobilization devices; that is, only a thermoplastic sheet with a flat pelvic base plate is used. Although the shifts measured by using CBCT are smaller than those in OPI, the large shifts measured by using OPI had been nullified. Thus, the results obtained by using CBCT cannot be compared with the results obtained by using OPI in this study or the results of other studies already published on imaging by using the OPI or CBCT technique for prostate cancer. However, the shift measured by using CBCT even after OPI is due to the interfractional and intrafractional motions of the prostate according to the position of the pelvic bones, 5,16 and this daily shift cannot be corrected by using bony landmarks based on OPI.

Although the doses may be slightly higher in the case of CBCT, the purpose of CBCT is image guidance, which has 
its benefits. However, Walter et al.17 indicated that onetime CBCT of the pelvic region delivers $17.2 \mathrm{mGy}$ to the rectum, $\leq 28 \mathrm{mGy}$ to the surface, and $10.2 \mathrm{mGy}$ to the center of the body, and the peripheral computed tomography dose index (CTDI) is $23.6 \mathrm{mGy}$. However, if it is calculated for $38 \#$, then the dose will remain at 65.36 cGy to the rectum, $\leq 106.4$ cGy to the surface, and 38.76 cGy to the center, and the CTDI will be 89.68 cGy. These values are in $38 \#$ and hence are negligible if compared with the pelvic dose tolerance values for OAR.

Since CBCT as a position verification tool is more time consuming, daily imaging with the OPI technique and performing CBCT once or twice a week can improve the precision and effectiveness of prostate cancer treatment. ${ }^{18}$ Table 1 shows that the mean IDV with CBCT was below $0.5 \mathrm{~cm}$ for five patients but $>0.5 \mathrm{~cm}$ for another five patients, showing that the prostate shift according to the position of the pelvic bones varies between patients. In addition, Figure 2 clearly indicates that the prostate shift in all three directions, namely the vertical, longitudinal, and lateral directions, is low for imaging sessions 1 to 25 (i.e., patient No. 1), 125 to 150 (i.e., patient No. 6), and 200 to 250 (i.e., patient Nos. 9 and 10) but high (specially longitudinal shift) for imaging sessions 150 to 200 (i.e., patient Nos. 7 and 8). A major factor along with the other reasons for such kind of shift is the rectum and bladder filling during the radiotherapy. ${ }^{19}$ Moiseenko et al. ${ }^{20}$ concluded in their study that bladder volume does not affect prostate shift and PTV underdosing / overdosing but that daily variation in bladder volume causes the undesirable dose delivery to it. Thus, in general, if the volumes of the rectum and bladder are maintained throughout the radiotherapy procedure while performing CT for the patients before planning and positioning them on the Clinac couch for each treatment session, then the prostate shift and doses to the OAR can be managed as per the planning performed on TPS. However, many patients cannot cooperate due to their own clinical problems; hence, daily CBCT becomes very important in such cases. ${ }^{21}$

\section{Conclusion}

Imaging with CBCT provides more-accurate prostate localization than the OPI technique in prostate cancer cases. In this study, even after bone-to-bone matching by OPI, a significant shift in the prostate was observed when position was cross-verified by CBCT.CBCT should be chosen as the preferred imaging technique. By using this imaging technique, tighter margins at the prostate-rectal interface can be used, thus preventing an undesirably high dose to the rectum and bladder.

\section{Conflict of interest}

The authors declare that they have no conflicts of interest. The authors alone are responsible for the content and writing of the paper.

\section{Acknowledgement}

We would like to express our thanks to director, Roentgen Oncologic Solutions Pvt. Ltd. and Chairman, Sri Aurobindo Institute of Medical Sciences for permitting and encouraging the study. We would also like to express our appreciation for the radiotherapy technologists Rupesh Bornare, Ramesh Kumar and Shailesh Patel for their support.

\section{References}

1. World Health Organization. World Cancer Report 2014. World Health Organization: Geneva; 2014.

2. Isa M, Rehman J, Afzal M, Chow JC. Dosimetric dependence on the collimator angle in prostate volumetric modulated arc therapy. Int J Cancer Ther Oncol. 2014;2:020419.

3. Rana S, Larson G, Vargas C, et al. Intensity modulated proton therapy versus uniform scanning proton therapy: Treatment planning study of the prostate cancer in patients with a unilateral metallic hip prosthesis. Jour Proton Ther. 2015;1:113.

4. Sawant S, Shegokar R. Cancer research and therapy: Where are we today? Int J Cancer Ther Oncol. 2014; 2:02048.

5. Wong JR, Gao Z, Uematsu M, et al. Interfractional prostate shifts: review of 1870 computed tomography (CT) scans obtained during image-guided radiotherapy using CT-on-rails for the treatment of prostate cancer. Int J Radiat Oncol Biol Phys. 2008;72:1396-401.

6. Hunt MA, Schultheiss TE, Desobry GE, et al. An evaluation of setup uncertainties for patients treated to pelvic sites. Int J Radiat Oncol Biol Phys. 1995;32:227-33.

7. Bentel GC, Marks LB, Sherouse GW, et al. The effectiveness of immobilization during prostate irradiation. Int J Radiat Oncol Biol Phys. 1995;31:143-8.

8. Russo GA, Qureshi MM, Truong MT, et al. Daily orthogonal kilovoltage imaging using a gantry-mounted on-board imaging system results in a reduction in radiation therapy delivery errors. Int J Radiat Oncol Biol Phys. 2012;84:596-601.

9. Gurjar OP, Mishra SP, Bhandari V, et al. A study on the necessity of $\mathrm{kV}$-CBCT imaging compared to $\mathrm{kV}$-Orthogonal portal imaging based on setup errors: Considering other socioeconomical factors. J Can Res Ther. 2014;10:583-6.

10. Ullman KL, Ning H, Susil RC, et al. Intra-and inter-radiation therapist reproducibility of daily isocenter verification using prostate fiducial markers. Radiat Oncol. 2006;1:2. 
11. Sveistrup J, af Rosenschöld PM, Deasy JO, et al. Improvement in toxicity in high risk prostate cancer patients treated with image-guided intensity-modulated radiotherapy compared to 3D conformal radiotherapy without daily image guidance. Radiat Oncol. 2014;9:44.

12. ICRU Report 83. Prescribing, recording, and reporting photon-beam intensity-modulated radiation therapy (IMRT). International Commission on Radiation Units and Measurements, Bethesda; 2010.

13. Mechalakos JG, Hunt MA, Lee NY, et al. Using an onboard kilovoltage imager to measure setup deviation in intensity-modulated radiation therapy for head-and-neck patients. J Appl Clin Med Phys. 2007;8:2439.

14. Zelefsky MJ, Crean D, Mageras GS, et al. Quantification and Predictors of prostate position variability in 50 patients evaluated with multiple CT scans during conformal radiotherapy. Radiother Oncol. 1999; 50:225-34

15. Barney BM, Lee RJ, Handrahan D, et al. Image-guided radiotherapy (IGRT) for prostate cancer comparing $\mathrm{kV}$ imaging of fiducial markers with cone beam computed tomography (CBCT). Int J Radiat Oncol Biol Phys. 2011;80:301-5.

16. Aubry JF, Beaulieu L, Girouard LM, et al. Measurements of intrafraction motion and interfraction and intrafraction rotation of prostate by three-dimensional analysis of daily portal imaging with radiopaque markers. Int J Radiat Oncol Biol Phys. 2004;60:30-9.

17. Walter C, Boda-Heggemann J, Wertz H, et al. Phantom and in-vivo measurements of dose exposure by image-guided radiotherapy (IGRT): MV portal images vs. kV portal images vs. cone-beam CT. Radiother Oncol. 2007; 85:418-23.

18. Adamczyk M, Piotrowski T, Adamiak E. Evaluation of combining bony anatomy and soft tissue position correction strategies for IMRT prostate cancer patients. Rep Pract Oncol Radiother. 2012;17:104-9.

19. Schild SE, Casale HE, Bellefontaine LP. Movements of the prostate due torectal and bladder distension: implications for radiotherapy. Med Dosim. 1993;18:13-5.

20. Moiseenko V, Liu M, Kristensen S, et al. Effect of bladder filling on doses to prostate and organs at risk: a treatment planning study. J Appl Clin Med Phys. 2006;8:55-68.

21. Sresty NV, Ramanjappa T, Raju AK, et al. Optimal usage of cone beam computed tomography system with different field of views in image guided radiotherapy (IGRT). Int J Cancer Ther Oncol. 2015; 3:334. 